\title{
Adjustment of successive and simultaneous intervals by musically experienced and inexperienced subjects
}

\author{
J. ELLIOT, J. R. PLATT, and R. J. RACINE \\ McMaster University, Hamilton, Ontario, Canada
}

\begin{abstract}
A single-stimulus adjustment technique was used to test the accuracy with which musically experienced and inexperienced subjects could intone musical intervals. We were particularly interested in the relationship between any improved accuracy resulting from musical training and interval properties, such as size or musical consonance, that could provide clues as to the mechanism of the improvement. The range of adjustment was restricted to a single chromatic interval to obviate the need for categorical interval labels. The intervals were selected so that the roles of both interval size and musical consonance could be tested. Simultaneous (harmonic) and successive (melodic) intervals, composed of both simple and complex tones, were examined. The experienced subjects were generally more accurate in their mean settings, although there was a slight tendency to stretch the smaller intervals. The inexperienced subjects tended to compress all intervals, expecially the larger ones. The experienced subjects were also more consistent in their settings, with the degree of consistency relating directly to the musical consonance of the interval. The inexperienced subjects, on the other hand, were also affected by absolute interval size and sensory consonance, displaying more consistency for smaller intervals and with simultaneous complex tones.
\end{abstract}

The perception of isolated musical intervals has been extensively investigated, and a recent review has been provided by Burns and Ward (1982). The most commonly used techniques have been identification and adjustment. The former technique requires that the subject provide chromatic labels for specified auditory intervals, whereas the latter requires the subject to adjust an auditory interval to match a specified chromatic label. In both cases it is assumed that the subject has previously acquired a standard set of verbal labels for the intervals of the chromatic scale. Consequently, these techniques are not applicable to musically inexperienced subjects. Even in the case of musically trained subjects, it is not clear to what extent performance is affected by perception of the auditory stimuli, as opposed to confusion about the relationship between the stimuli and their labels. Any attempt to examine the effects of musical experience on the perception of isolated intervals requires a technique that does not assume the prior acquisition of such a set of labels.

In the experiment reported here, we used a variation of the adjustment technique. In the usual adjustment experiment (e.g., Moran \& Pratt, 1926), the experimenter names a chromatic interval (e.g., "perfect fifth") and provides the subject with two tones, one of fixed frequency and one variable. The subject then adjusts the variable tone until its interval relationship to the fixed tone matches that specified. It is assumed (Burns \& Ward, 1982) that

This research was supported by Grant A8269 from the Natural Sciences and Engineering Research Council of Canada. Reprints may be obtained from the second author at the Department of Psychology, McMaster University, 1200 Main Street West, Hamilton, ON L8S 4K1, Canada. the label accesses an internal standard against which the subject makes his/her adjustments, and that the only role of the verbal label is to cue which standard is to be used. The need for this cue could be eliminated if the allowable range of adjustment spanned no more than one label. This was accomplished in the present experiment by limiting the range of adjustment of the interval to $\pm^{1 / 2}$ semitone from the nominal interval of interest.

The variable of primary interest was the musical experience of the subjects. If musical intervals constitute a basic alphabet for musical expression, the means by which the internal standards for these intervals are acquired is an important question for those interested in the mechanisms underlying music skills. A first step in this direction is determination of possible differences in these internal standards between experienced and inexperienced subjects. We also manipulated some of the variables that had already been implicated in the perception of isolated musical intervals by experienced subjects. It is possible, for example, that the internal standards are acquired in order of musical consonance, with the most consonant intervals learned first or more accurately. It is also possible that these intervals are learned in order of interval size, with smaller intervals learned more rapidly than larger ones. When notes are presented simultaneously (i.e., harmonically, as in chords), musically consonant intervals are heard more frequently than are less consonant intervals. During successive (melodic) presentation, on the other hand, smaller intervals tend to occur more frequently than larger ones (Jeffries, 1974). Consequently, we also manipulated the method of presentation (simultaneous vs. successive) of the intervals. Finally, we used both sim- 
ple and complex tones, because complex tones provide increased information based on sensory consonance of overtones when presented simultaneously. Resulting adjustments were examined for intrasubject variability and mean value to determine whether, and, more importantly, in what way, the performance of musically experienced subjects was superior to that of inexperienced subjects.

\section{METHOD}

\section{Subjects}

The subjects were 32 volunteers recruited from an introductory psychology course. Sixteen were classified as musically experienced on the basis of self-reports of at least 3 years of formal instruction on a musical instrument other than percussion. Reported years of instruction ranged from 3 to 13 . The other 16 subjects reported no instrumental instruction and were classified as musically inexperienced. One subject was lost from the musically inexperienced group due to experimenter error.

\section{Apparatus}

Synthesis and presentation of auditory stimuli, as well as recording of responses, were controlled by a Yamaha CX5M Music Computer. Synthesized auditory stimuli were conditioned by a Panasonic RX-C60 amplifier and presented binaurally to the subjects through Realistic Pro-1 earphones. Subjects indicated their responses using the cursor-control keys and the space bar on the computer keyboard.

\begin{abstract}
Stimuli
Simple tones were sine waves with 50 -msec rise and fall times. Complex tones were produced by a FM-synthesized acoustic piano (Voice Number 8 from the CX5M's internal ROM). All tones were set at $75 \mathrm{~dB}$ SPL, and had a duration of $500 \mathrm{msec}$. The fixed tone for all intervals was $440 \mathrm{~Hz}$. The nominal values of the variable tones were defined by the frequency ratios of A-major just intonation to be $9 / 8$ or $495 \mathrm{~Hz}$ for the major second, $4 / 3$ or $586.6 \mathrm{~Hz}$ for the perfect fourth, $3 / 2$ or $660 \mathrm{~Hz}$ for the perfect fifth, $15 / 8$ or $825 \mathrm{~Hz}$ for the major seventh, and $2 / 1$ or $880 \mathrm{~Hz}$ for the octave. The definition of the tritone for just intonation is ambiguous. The present study followed Roederer (1979) in using a frequency ratio of $45 / 32$ to obtain $618.75 \mathrm{~Hz}$. Each trial began with the frequency of the variable tone randomly set according to a uniform distribution ranging from -25 to +25 cents of its nominal frequency. It could then be adjusted over a range of minus to plus 50 cents of the nominal frequency.
\end{abstract}

\section{Procedure}

Each subject participated in two sessions, lasting approximately $1 \mathrm{~h}$ each, with at least 1 day between sessions. A session consisted of 48 trials, 8 with each interval size. The order of interval sizes was randomized within blocks of 8 trials. In one session both tones composing the interval were presented simultaneously (harmonically) with $1 \mathrm{sec}$ of silence between presentations. In the other session the two tones were presented successively (melodically), in ascending order, with $500 \mathrm{msec}$ of silence between the two tones and $1 \mathrm{sec}$ between intervals. The order of the harmonic and melodic sessions was counterbalanced. Although all subjects were tested on both melodic and harmonic intervals, they were tested on only one of the tone types. Half of the subjects from each musical experience group received only simple tones during the two sessions, whereas the other half received only complex tones.

The subjects were instructed to adjust the pitch of the variable tone until it "harmonized best" with the fixed tone. Adjustments could be made at any time using the cursor-control keys. The "up" and "down" keys increased and decreased, respectively, the frequency of the variable tone in 10-cent increments. The "right" and "left" keys increased and decreased, respectively, this frequency in 1-cent increments. The subjects could make as many adjustments as they liked, but the effect was not heard until the next presentation of the interval. The variable tone could not be adjusted more than \pm 50 cents of its nominal frequency, and any attempt to do so produced a warning on the video monitor. The subjects indicated their satisfaction with the adjusted interval by pressing the space bar on the computer keyboard, which terminated the trial and began a new one. All subjects were observed for any tendency to adjust the variable tone to an extreme and then readjust it a fixed number of steps in the other direction. Such a pattern did not occur.

\section{RESULTS}

To facilitate evaluation in a musical context, each final setting of a variable stimulus was converted from hertz to cents' deviation from the nominal, justly tempered interval. Each subject's performance on each trial type was then summarized by two measures. Relative error was the standard deviation of the settings and constant error was their mean.

An analysis of variance of mean relative errors yielded significant effects of musical experience $[F(1,27)=$ $36.77, p<.001]$ and of interval size $[F(5,135)=11.06$, $p<.001]$, as well as a significant interaction of these variables $[F(5,135)=3.32, p<.01]$. The left panel of Figure 1 shows mean relative errors for the two groups across the various interval sizes. Experienced subjects were more consistent in their interval settings than were inexperienced subjects. An interval is usually considered to be musically consonant to the extent that the ratio of its frequencies can be reduced to a ratio of small integers. The right panel of Figure 1 replots the intervals in decreasing order of musical consonance by reference to the magnitude of the smallest integer ratio to which the interval can be reduced. It is clear that the consistency with which experienced subjects adjusted the interval increased with musical consonance. Although interval size had a less pronounced effect on the consistency of settings by inexperienced subjects, a planned comparison showed it to be significant $[F(5,135)=2.52, p=.032]$. This effect for inexperienced subjects was not totally determined by musical consonance of the intervals, but also appeared to reflect a tendency to be more consistent for smaller intervals.

In addition to the effects of musical experience and interval size, relative errors also displayed several significant effects involving method of presentation (simultaneous vs. successive) and tone type (simple vs. complex). There was a significant effect of method of presentation $[F(1,27)=4.20, p=.048]$ and a significant interaction of this variable with tone type $[F(1,27)=5.88, p=.021]$. This was due to greater consistency in the adjustments of harmonic intervals made up of complex tones. The mean relative error for these intervals was only 16.4 cents. Comparable values were 19.5, 19.7, and 

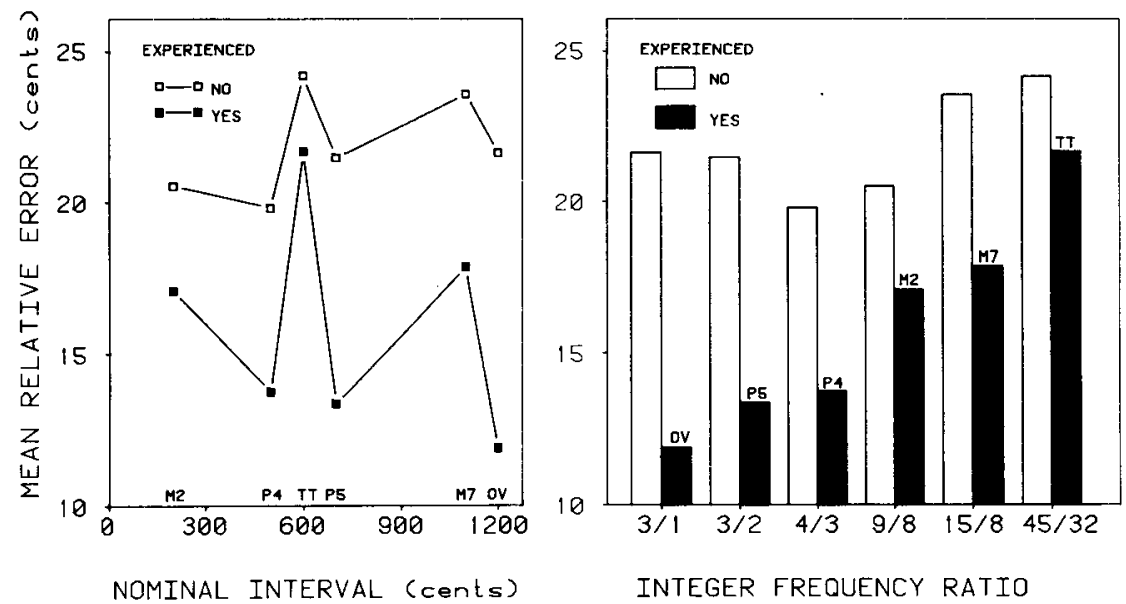

Figure 1. Mean relative error of adjustment, in cents, for musically experienced and inexperienced subjects on the various intervals. The left panel plots the data against absolute interval width; the right panel arranges the intervals in decreasing order of musical consonance, as indexed by the smallest integer ratio to which the interval could be reduced.

19.9 cents for melodic presentation of complex tones and harmonic and melodic presentation of simple tones, respectively.

There was also a significant interaction of method of presentation and interval size $[F(5,135)=5.98$, $p<.001]$, which in turn interacted with musical experience $[F(5,135)=2.97, p=.014]$. Table 1 shows the means for this three-way interaction. Musically inexperienced subjects set simultaneous intervals more consistently than successive ones, except for a reversal of this effect at the major second. Experienced subjects set successive intervals more consistently at the major second and perfect fourth and simultaneous intervals more consistently at the tritone and perfect fifth, and showed very little difference between methods of presentation at the major seventh and octave. It should also be noted that the experienced subjects were particularly inconsistent with successive presentation of the tritone, performing no better than musically inexperienced subjects.

An analysis of variance of mean constant errors yielded significant effects of musical experience $[F(1,27)=$ $10.62, p<.01]$ and of interval size $[F(5,135)=2.62$, $p=.027]$. No other main effects or interactions approached statistical significance. Figure 2 shows mean constant errors for the two groups across the various interval sizes. Inexperienced subjects tended to set intervals narrower than their nominal value, and this tendency increased with the width of the interval, with a slight reversal in the trend at the octave. Experienced subjects showed a similar trend toward narrower interval settings (compared with the just standard) as interval width increased, but they set all intervals wider than did inexperienced subjects. The experienced subjects, in fact, set the three smallest intervals (M2, P4, and TT) slightly wider than their nominal value.

\section{DISCUSSION}

Relative errors for musically experienced subjects were in agreement with those obtained by identification and adjustment techniques involving the use of chromatic labels. The increase in consistency of settings with musical consonance of the interval is comparable to the adjustment results of Moran and Pratt (1926), as well as to Killam, Lorton, and Schubert's (1975) finding of greater accuracy of identification of more musically consonant intervals. The absolute values of the relative errors were also similar to others reported in the literature. For example, Burns

Table 1

Mean Relative Errors in Cents as a Function of Musical Experience, Method of Presentation, and Nominal Interval Width

\begin{tabular}{|c|c|c|c|c|c|c|c|}
\hline \multirow[b]{2}{*}{ Experience } & \multirow[b]{2}{*}{$\begin{array}{l}\text { Method of } \\
\text { Presentation }\end{array}$} & \multicolumn{6}{|c|}{ Nominal Interval } \\
\hline & & $\begin{array}{l}\text { Major } \\
\text { Second }\end{array}$ & $\begin{array}{l}\text { Perfect } \\
\text { Fourth }\end{array}$ & Tritone & $\begin{array}{l}\text { Perfect } \\
\text { Fifth }\end{array}$ & $\begin{array}{c}\text { Major } \\
\text { Seventh }\end{array}$ & Octave \\
\hline No & $\begin{array}{l}\text { Successive } \\
\text { Simultaneous }\end{array}$ & $\begin{array}{l}18.8 \\
22.3\end{array}$ & $\begin{array}{l}21.3 \\
18.3\end{array}$ & $\begin{array}{l}25.0 \\
23.3\end{array}$ & $\begin{array}{l}22.8 \\
20.2\end{array}$ & $\begin{array}{l}25.4 \\
21.7\end{array}$ & $\begin{array}{l}25.1 \\
18.1\end{array}$ \\
\hline Yes & $\begin{array}{l}\text { Successive } \\
\text { Simultaneous }\end{array}$ & $\begin{array}{l}14.4 \\
19.8\end{array}$ & $\begin{array}{l}12.2 \\
15.3\end{array}$ & $\begin{array}{l}25.4 \\
18.0\end{array}$ & $\begin{array}{l}15.2 \\
11.6\end{array}$ & $\begin{array}{l}17.2 \\
18.5\end{array}$ & $\begin{array}{l}12.5 \\
11.3\end{array}$ \\
\hline
\end{tabular}




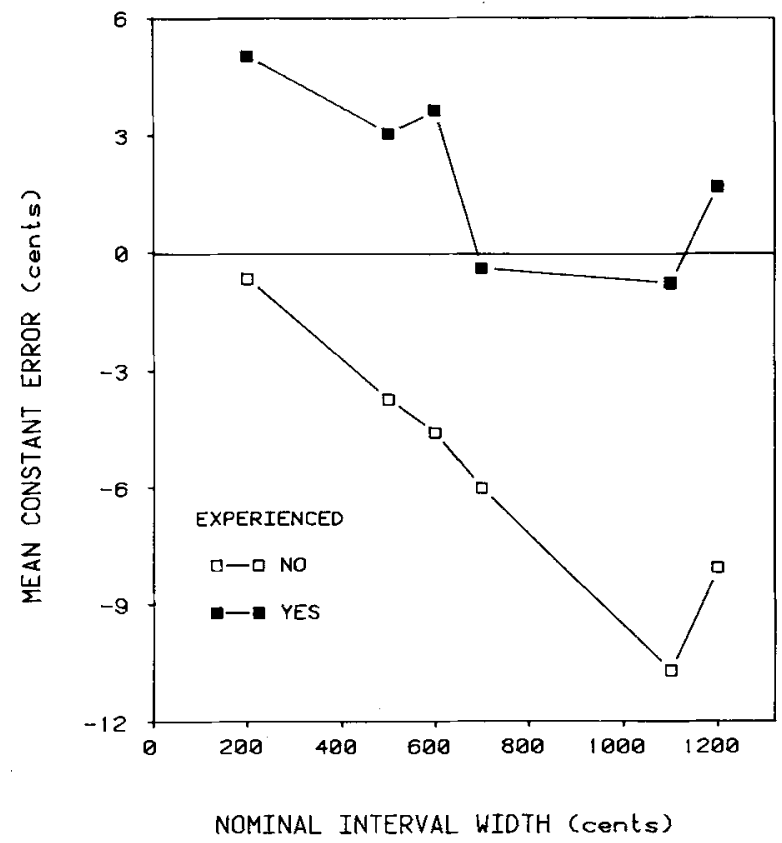

Figure 2. Mean constant error of adjustment, in cents, for musically experienced and inexperienced subjects as a function of nominal interval width.

and Ward (1982) pointed out that the mean standard deviation of repeated adjustments of sequential or simultaneous octaves composed of simple tones is on the order of 10 cents (Terhardt, 1969; Ward, 1953, 1954) and is slightly lower for complex tones (Sundberg \& Lindquist, 1973; Terhardt, 1969; Walliser, 1969). Comparable values for musically experienced subjects in the present experiment were 12.4 and 11.4 cents, respectively.

The present experiment allowed evaluation of the effects of musical experience. The interval settings of musically inexperienced subjects were less consistent than those of experienced subjects, and were less consistently affected by the musical consonance of the interval being adjusted. The small effect of interval size on relative errors for inexperienced subjects may be due to the differences in frequency of exposure to these intervals while listening to music. The case for a familiarity effect is even stronger for the musically experienced subjects. Not only did they produce settings that were more consistent overall, but their degree of superiority over inexperienced subjects was predictable from the musical consonance of the nominal interval being adjusted. Although there is a historical trend to increasing frequencies and ranges of dissonances, Western harmony has traditionally used the more musically consonant harmonic intervals more often than the less consonant ones (Dowling \& Harwood, 1986). This frequency effect may be most dramatically illustrated in the present results by the fact that experienced subjects were no more consistent than inexperienced subjects in adjusting sequentially presented tritones, since this nondiatonic interval is rarely used, either harmonically or melodically, in traditional Western music.

In addition to the role of familiarity of musical intervals, the relative errors of inexperienced subjects also suggest the operation of sensory consonance. Sensory consonance refers to the rated "pleasantness" of pairs of tones presented simultaneously in isolation. Such ratings are determined by the general pattern of interaction between their pure-tone components in the auditory system, as described by the critical bandwidth (Terhardt, 1984). In the case of simple tones (Plomp \& Levelt, 1965), sensory consonance ratings decline sharply from the unison to a minimum at about one-quarter of a critical bandwidth, after which they increase and are uniformly high for intervals greater than the critical bandwidth. The picture is similar for complex tones (Kameoka \& Kuriyagawa, 1969), except that there are also local maxima at simple integer ratios of the two fundamental frequencies, attributable to the maximum coincidence of harmonics of the two tones under these circumstances. As Terhardt (1984) indicated, sensory consonance does not completely account for musical consonance, inasmuch as ratings of the former are far from perfectly correlated with indices of the latter obtained from analyses of tonal music.

All intervals in the present study were in excess of the critical bandwidth, except for the major second, which represents approximately one-half of the critical bandwidth. The overall greater consistency of adjustments of simultaneous intervals of complex tones almost certainly reflects the role of sensory consonance in this condition. Only in this condition would there be local maxima of sensory consonance at the justly intoned intervals to augment whatever internal standard controlled the subjects' adjustments. In addition, a closer examination of the generally greater consistency with which inexperienced subjects adjusted simultaneous, as opposed to successive, intervals indicated that the effect was almost entirely attributable to those subjects who received complex tones. This suggests that they relied on tonal consonance in the absence of well-developed internal standards for musical intervals. The reversal of this effect at the major second would be expected, because the simultaneous presentation of this interval of less than a critical bandwidth sounds rough and discordant no matter how it is adjusted. The experienced subjects were less affected by whether the intervals were simultaneous or successive, which probably reflects a better developed set of internal standards for musical intervals and a decreased reliance on sensory consonance.

The constant errors obtained in the present study were small, with mean values within about 10 cents of the nominal interval. It is unlikely that this was due simply to the small range of adjustment allowed. The errors could have approached \pm 50 cents, even allowing for some avoidance of the ends for the adjustment range. There was a general tendency for constant errors to shift in a negative direction as interval size increased, with a slight reversal in this trend at the octave. Although small, this trend 
is in the direction opposite to that usually found in studies in which the adjustment response is cued with a chromatic label (Burns \& Ward, 1982). Such studies have generally reported a tendency to "compress" smaller intervals and "stretch" wider intervals, perhaps reflecting a simple tendency to exaggerate adjustments in opposite directions as either end of a set of dimension labels is approached. This type of range effect would not have occurred in the present study because chromatic labels were not employed. It is not clear why a small trend in the opposite direction was found, but the present results do show that the pattern of constant errors usually obtained may have more to do with the use of chromatic labels than with the perception of the auditory intervals.

The other major aspect of the constant-error results was a tendency for constant errors to be slightly positive for musically experienced subjects, but predominately negative for inexperienced subjects. The experienced subjects' result may be another instance of musicians' general tendency to perform with sharp intonation (Ward, 1970) and to judge just intervals to sound flat (Terhardt \& Zick, 1975). Comparable data are not available for musically inexperienced subjects, but the present findings suggest that sharp intonation is probably a product of musical training, rather than a primary characteristic of the auditory system.

A final consideration concerning the present results is their relation to categorical perception. A number of studies reviewed by Burns and Ward (1982) attempted with some success to show that continuous variations in interval sizes may be perceived discretely, in accordance with chromatic pitch labels. Some authors (e.g., Siegel \& Siegel, 1977) have even suggested that musicians may not be able to discriminate between sharp and flat intonations within such a chromatic category. If this were strictly true, the ability of subjects in the present study to produce nonrandom adjustments of intervals whose range of adjustment was restricted to a single chromatic category would be very suspect. However, Wapnick, Bourassa, and Sampson (1982) showed that although musicians respond categorically to intervals presented both in isolation and in melodic context, they can discriminate between sharp and flat intonations within a category at considerably better than chance levels.

In this context, the present study avoided the issue of categorical perception of intervals, and a labeling system for these categories, by restricting the range of adjustment of an interval to a single chromatic category. It was then possible to examine the extent to which both musically experienced and inexperienced subjects have an internal standard that would support consistent intonation of the interval. The results indicated not only that such standards and their utilization were better developed in musically experienced subjects, but that this advantage was strongly related to the musical consonance of the interval, suggesting the importance of familiarity with the interval being adjusted. In addition, the results implicated sensory consonance in the adjustment of simultaneous intervals of complex tones, particularly in the case of musically inexperienced subjects.

\section{REFERENCES}

Burns, E. M., \& WARD, W. D. (1982). Intervals, scales, and tuning. In D. Deutsch (Ed.), The psychology of music. New York: Academic Press.

Dowling, W. J., \& Harwood, D. L. (1986). Music cognition. New York: Academic Press.

JEFFrIES, T. B. (1974). Relationship of interval frequency count to ratings of melodic intervals. Journal of Experimental Psychology, 102, 903-905.

Kameoka, A., \& KuriYagawa, M. (1969). Consonance theory: Part II. Consonance of complex tones and its calculation method. Journal of the Acoustical Society of America, 45, 1460-1471.

Killam, R. N., LorTon, P. V., \& SchuberT, E. D. (1975). Interval recognition: Identification of harmonic and melodic intervals. Journal of Music Theory, 19, 212-234.

Moran, H., \& Pratt, C. C. (1926). Variability of judgements of musical intervals. Journal of Experimental Psychology, 9, 492-500.

Plomp, R., \& LeVelt, W. J. M. (1965). Tonal consonance and critical band-width. Journal of the Acoustical Society of America, 35, 548-560.

ROEDERER, J. G. (1979). Introduction to the physics and psychophysics of music (2nd ed.). New York: Springer-Verlag.

Siegel, J. A., \& Siegel, W. (1977). Categorical perception of tonal intervals: Musicians can't tell sharp from flat. Perception \& Psychophysics, 21, 399-407.

SUNDBERG, J., \& LINDQUIST, J. (1973). Musical octaves and pitch. Jour nal of the Acoustical Society of America, 54, 922-927.

TERHARDT, E. (1969). Oktavspreizung und Tonhöhen der Schiefbung bei Sinustonen. Acustica, 22, 348-351.

TERHARDT, E. (1984). The concept of musical consonance: A link between music and psychoacoustics. Music Perception, 1, 276-295.

TerHARDT, E., \& ZICK, M. (1975). Evaluation of the tempered tone scale in normal, stretched and contracted intonation. Acustica, 32, 268.

WALLISER, U. (1969). Über die Spreizung von empfundenen Intervallen gegenüber mathematisch harmonischer Intervallen bei Sinustones. Frequenzy, 23, 139-143.

WaPNick, J., Bourassa, G., \& SAmpson, J. (1982). The perception of tonal intervals in isolation and in melodic context. Psychomusicology, 2, 21-37.

WARD, W. D. (1953). The subjective octave and the pitch of pure tones. Unpublished doctoral dissertation, Harvard University, Cambridge, MA.

WARD, W. D. (1954). Subjective musical pitch. Joumal of the Acoustical Society of America, 26, 369-380.

WARD, W. D. (1970). Musical perception. In J. V. Tobias (Ed.), Foundations of modern auditory theory (Vol. 1, pp. 405-447). New York: Academic Press.

(Manuscript received January 27, 1987; revision accepted for publication May 28,1987 .) 